dermatitis, their skin condition were evaluated by a dermatologist/allergologist and by TEWL (trans-epidermal water loss) measurements. Information on skin disease, occupational and non-occupational exposure were recorded using a standardized questionnaire. Workers participated to a second control after 3 months, to verify the effectiveness of the training.

Results 80 workers with an occupational hand dermatitis accepted to participate to a training course and 62 (77.5\%) completed the 3 months follow-up. Symptoms improved after the training for the $73 \%$ of subjects that adhered to suggestions and followed recommendations and for the $38 \%$ of workers that partially followed recommendations $(p<0.01)$. TEWL values improved at the end of follow-up, confirming the positive effect of protective measurements suggested on skin barrier function.

Discussion Our intervention was effective, leading to a reduction in clinical signs of dermatitis in people that strictly adhere to recommendations, mainly on cleaning habits and skin cream use. The educational intervention permitted to improve skin condition in a short-term follow-up. More efforts are needed to train workers on skin protection procedures.

\section{8d SCIN TRIAL (SKIN CARE INTERVENTION IN NURSES)}

Ira Madan. Guy's and St Thomas NHS Foundation Trust, London, UK

\subsection{6/oemed-2018-ICOHabstracts.260}

Introduction The high prevalence of hand dermatitis in nurses is attributed to frequent hand washing with soap and infrequent use of hand moisturisers. We tested the hypothesis that a behavioural change programme (BCP) coupled with hand moisturisers, can reduce the prevalence of hand dermatitis in at-risk nurses over a 12 - month period.

Methods We conducted a cluster randomised design at 35 sites. We recruited two groups of at-risk nurses:

a. first year student nurses with a history of atopic tendency;

b. intensive care unit (ICU) nurses.

The BCP was offered to participants at intervention plus sites only and participants were asked to form implementation intentions for performing each hand care behaviour in the workplace. Student nurses were provided with personal supplies of hand creams and ICU nurses encouraged to make use of the moisturisers on the wards. All participants, including those at intervention light sites, were provided with an advice leaflet on optimal hand care. Participants had their hands photographed at baseline and 12 months and these were objectively assessed by a dermatology research nurse and two dermatologists. We also measured changes in hand dermatitis, beliefs and behaviours and collected health economic data.

Results Overall the results indicate that the intervention had a positive (but not statistically significant at 5\% level) effect in reducing the prevalence of hand dermatitis compared to usual care and had a positive effect in changing behaviours and beliefs associated with dermatitis prevention. The BCP was also found to be of low cost to implement. (Please note, final results were not available by the abstract submission date but will be provided to the Secretariat in due course).

Discussion A hand-dermatitis prevention BCP plus optimal provision of hand moisturisers has been shown to be effective in reducing hand dermatitis in nurses. 1618e MINIMUM STANDARDS ON PREVENTION, DIAGNOSIS AND TREATMENT OF OCCUPATIONAL AND WORKRELATED SKIN DISEASES IN EUROPE - POSITION PAPER OF THE COST ACTION STANDERM (TD 1206)

${ }^{1} J o s e ́$ Hernán Alfonso, ${ }^{2}$ Patricia Weinert. ${ }^{1}$ Dept. of Occupational Medicine and Epidemiology, National Institute of Occupational Health, Oslo, Norway; ${ }^{2}$ Institute for interdisciplinary Dermatological Prevention and Rehabilitation (iDerm), Germany

\subsection{6/oemed-2018-ICOHabstracts.261}

Introduction Skin diseases constitute up to $40 \%$ of all notified occupational diseases in most European countries, predominantly comprising contact dermatitis, contact urticaria, and skin cancer. While insufficient prevention of work-related skin diseases (WRSD) is a top-priority problem in Europe, common standards for prevention of these conditions are lacking.

Objective To develop common European standards on prevention and management of WRSD and occupational skin diseases (OSD). Method Consensus amongst ca. 100 experts within occupational dermatology was achieved with regard to the definition of minimum evidence-based standards on prevention and management of WRSD/OSD (Delphi Technique).

Results By definition, WRSDs/OSDs are (partially or fully) caused by occupational exposure. The definition of OSD sensu stricto additionally includes diverging national legal requirements, with an impact on registration, prevention, management, and compensation. With the implementation of the classification of WRSD/OSD in the International Classification of Diseases (ICD) 11th Revision in future, a valid surveillance and comparability across countries will be possible. Currently, WRDS and OSD are still under-reported. Depending on legislation and regulations, huge differences exist in notification procedures in Europe, although notification is crucial to prevent chronic and relapsing disease. Facilities for early diagnosis, essential for individual patient management, should be based on existing guidelines and include a multidisciplinary approach. Patch testing is essential if contact dermatitis persists or relapses. Workplace exposure assessment of WRSD/OSD requires full labelling of product ingredients on material safety data sheets helping to identify allergens, irritants and skin carcinogens. Comparable standards in primary, secondary and tertiary prevention must be established in Europe to reduce the burden of WRSD/OSD in Europe.

Conclusion The adoption of common European standards on prevention of WRSD/OSD will contribute to reduce the incidence of OSD and their socio-economic burden.

\section{REFERENCE}

1. JEADV 2017;31(Suppl 4):1-13.

\section{Education and Training}

\section{HOW HEALTHY ARE MALE HEALTH SCIENCES STUDENTS AT IMAM ABDULRAHMAN BIN FAISAL UNIVERSITY?}

Sultan Al-Otaibi, Abullah Almutahhar, Taha Aljishi, Hussain Aljawad, Adulaiziz Sebiany. Imam Abdulrahman Bin Faisal University, Damam, Saudi Arabia

\subsection{6/oemed-2018-ICOHabstracts.262}

Objectives This study aimed to collect information on the personal health status of health sciences students in Imam Abdulrahman Bin Faisal University. It also aimed to investigate the 
relationships between levels of physical activity and other behaviours as well as specific health indicators in students attending college.

Methods A convenience sample of 147 college students, ages 18 to 24 years, participated in this research study at Imam Abdulrahman Bin Faisal University. The data were obtained through interview questionnaire and trained investigators collected weight, height, blood pressure and blood sugar measurements.

Results Forty-five percent of the students were either overweight or obese $(21.8 \%$ and $23.1 \%)$ while $17.0 \%$ of the students were current smokers and $7.5 \%$ of them were exsmokers. The majority of the students $(91.8 \%)$ had fast food (hamburger, French fries, and soft drink) and out of these $62.9 \%$ had it three times or more weekly. Furthermore, 35\% or more of the students did not have breakfast or drink milk or have vegetables on a daily basis. Also, 22.4\% of the students were inactive. Ten percent of the participants were hypertensive while $1.4 \%$ had impaired random blood sugar with increased risk for diabetes mellitus. All of these students were unaware that they had hypertension or high random blood sugar. There was a significant statistical relationship between living in-campus at university and frequency of consumption of fast food $(p=0.003)$.

Conclusion This study shows that there were high rates of risky lifestyles, namely, obesity, smoking, unhealthy diet, and inactivity among health sciences students, while some have health issues such as hypertension and high risk of diabetes.

\section{TRANSFÓRMATE: WORKPLACE HEALTH PROMOTION AND WELLNESS PROGRAM AT GRUPO PROGRESO}

\footnotetext{
1,2,30vidio Roberto Hermosilla Colmenares, 1,2,3 Zamarí Anabella López Hurtado. 'ISSA, Mining Section, Geneva, Switzerland; ${ }^{2} \mathrm{COH}$, Industrial Hygiene, Milan, Italy; ${ }^{3} \mathrm{ASOMET}$, Guatemalan Occupational Heath Association, Guatemala, Guatemala
}

\subsection{6/oemed-2018-ICOHabstracts.263}

Objective Reduce chronic non-communicable disease risk factors associated to poor diet and physical inactivity, plus increase knowledge about healthy lifestyles in employees at Grupo Progreso.

Methods Chronic diseases kill more than 38 million people every year, and almost three quarters of deaths occur in low and middle income countries. Metabolic syndrome is a cluster of risk factors that increase the risk to present cardiovascular disease and is becoming a public heath problem. In Latin American the prevalence is $25 \%$.

Metabolic syndrome is a set of 3 or more altered factors, for example:

Waist circumference, HDL cholesterol 'also known as good cholesterol', blood pressure, Triglycerides, Fasting glucose or pharmacotherapy for the last 3 factors. We decided to implement a wellness program called Transfórmate.

Labour intervention study in 1308 employees. We implemented a health promotion and wellness program, previously validated in a pilot project. Educational workshops were given where the main topics were: Dietary Guidelines for Guatemala, physical activity, pedometer program and stress management. All participants received personalised dietary treatment, practical sessions of physical activity, weekly text messaging, educational material, analysis of lipid profile, fasting glucose and drug treatment when needed. An assessment of initial and final knowledge was performed. Effectiveness in reducing risk factors for chronic non-communicable disease was calculated for people who had metabolic syndrome at the start of the program (N: 301). The data were analysed in STATA SE 13.0. measures of central tendency and dispersion were calculated. Hypothesis tests were performed using the Wilcoxon test for quantitative and McNemar Chi 2 for qualitative variables.

Results Of the 301 employees, 90.7\% was men and 9.3\% women with a median age of 40 (IQR 35-47). The set of strategies achieved a $47 \%$ reduction of the metabolic syndrome. Compared to other programs, in Korea they reported $30 \%$ of reduction and in Italy 35\%. The combination of educational, behavioural and biomedical interventions may explain this high percentage. The chronic non-communicable disease risk factors that reduced significantly were: low HDL-cholesterol 18\% ( $\mathrm{p} \leq 0.001)$, high triglycerides $14 \% \quad(\mathrm{p} \leq 0.001)$, low consumption of fruits and vegetables 13\% $(\mathrm{p} \leq 0.001)$ and physical inactivity $8 \%(\mathrm{p}=0.025)$. The median of daily steps were 8559 (IQR: 5,813-10,765), the median of the initial knowledge assessments were 70 (IQR: 50-80) and final 90 (RIC: 80-100).

Conclusion A significant reduction in metabolic syndrome and chronic non-communicable disease risk factors associated with an unhealthy diet and sedentary lifestyle was observed, in addition to an increase in knowledge about healthy lifestyles after implementing the program at the corporate level.

\section{DEVELOPMENT AND EVALUATION OF A TRAINING PROGRAMME ON SCIENTIFIC RESEARCH AND OCCUPATIONAL HEALTH SURVEILLANCE IN TURKEY}

${ }^{1} \mathrm{~L}$ Braeckman*, ${ }^{2} \mathrm{~A}$ Venema, ${ }^{2} \mathrm{~L}$ Hermans, ${ }^{2} \mathrm{~S}$ Adigüzel Van Zoelen, ${ }^{1} \mathrm{M}$ De Ridder, ${ }^{3} \mathrm{G}$ Van der Laan, ${ }^{3} \mathrm{~F}$ Van Dijk. 'Ghent University, Department of Public Health, Gent, Belgium; ${ }^{2}$ Applied Research Organisation TNO, Work and Health, Leiden, The Netherlands; ${ }^{3}$ Amsterdam Free University Medical Centre (VUmc), Department of Public Health, Amsterdam, The Netherlands

\subsection{6/oemed-2018-ICOHabstracts.264}

Introduction The general aim of the programme is to strengthen the occupational health expertise and scientific capacity of the Public Health Institution of Turkey (PHIT) by organising trainings, mentoring, exchange visits and networking.

Methods Following the ADDIE model (Analysis, Design, Development, Implementation and Evaluation), five training modules have been developed and implemented in the course of 2016. At present, we evaluate the training programme to see if the learning objectives have been met and to gather information in order to further enhance the efficiency and success rate of the project. Qualitative evaluation forms questioning the content and format of the trainings, the organisation and the trainers were filled in by the 44 participants from PHIT after each training module. Trainees also rated quantitatively on a scale from 0 to 10 , all parts of the training. To measure changes in knowledge and skills regarding occupational health surveillance and research, a self-efficacy questionnaire was developed and administered three times. Descriptive statistics were computed.

Results Overall, very high rankings and positive comments were generated. No major recommendations for improvement of the trainings were formulated. Some minor issues concerned the amelioration of the learning environment and conditions e.g. a better time management to assimilate the new 